\title{
TUBERCULOSIS KNOWLEDGE AMONG INJECTING DRUG USERS VISITING SYRINGE EXCHANGE PROGRAMME IN TALLINN, ESTONIA
}

\author{
Kristi Rüütel', R. David Parker², Igor Sobolev ${ }^{3}$, Helle-Mai Loit ${ }^{1}$ \\ ${ }^{1}$ National Institute for Health Development, Tallinn, Estonia \\ 2University of South Carolina, Columbia, SC, USA \\ ${ }^{3}$ NGO Estonian Network of People Living with HIV, Tallinn, Estonia
}

\begin{abstract}
SUMMARY
Background: The purpose of the current study was to describe tuberculosis (TB) knowledge, beliefs, and experience with TB services among injecting drug users.

Methods: Participants for this anonymous, cross-sectional study were recruited from a community based syringe exchange programme in Tallinn, Estonia. A structured questionnaire was completed and included information on socio-demographics, health history, drug use, and knowledge about TB and HIV.

Results: The study included 407 people ( $79 \%$ male, mean age 27.9 years, mean injection drug use 9.4 years). $32.9 \%$ of participants reported HIV infection and $1.7 \%$ lifetime history of TB. $26.4 \%$ participants $(n=106)$ reported symptoms suggestive of TB. $93 \%$ of participants recognized correctly that TB is air-borne infection and $91 \%$ that HIV is a risk factor for TB. Only $40 \%$ of the participants knew that TB diagnostics and treatment in Estonia are free of charge for everybody and $58 \%$ reported they knew where to get health care services in case they suspected that they had TB. TB transmission and treatment adherence knowledge was better among those in contact with either health care or harm reduction services, e.g the community based syringe exchange programme.

Conclusion: Similar to HIV services, TB prevention and education should be integrated into harm reduction and drug treatment programmes to facilitate early diagnosis and treatment of TB among injecting drug users.
\end{abstract}

Key words: injecting drug users, tuberculosis, HIV, harm reduction, syringe exchange

Address for correspondence: K. Rüütel, National Institute for Health Development, 42 Hiiu St, Tallinn 11619, Estonia. Email: kristi.ruutel@tai.ee

\section{INTRODUCTION}

Tuberculosis (TB) has re-emerged as a significant problem in Europe, partly because of poor TB control programmes and partly because of the link with HIV infection, migrants and other vulnerable populations (1). Illegal drug use has become a risk factor for TB as a result of the overlap of epidemiological and social factors associated with both drug use and TB (2). The dissemination of HIV infection among drug users, knowing that HIV is the most potent risk factor for TB disease progression among adults, makes drug users a critical risk-group and a target for TB screening (3).

In the last decade, Estonia has experienced a surge in HIV infections associated with injection drug use $(4,5)$. The incidence of TB was 26 per 100,000 in 2008 and $9 \%$ of TB cases were also HIV-positive ( $\mathrm{n}=37$ ). The first HIV-TB co-infection case was diagnosed in 1997 and the numbers have increased annually (6). The overlap of the HIV and injecting drug use (IDU) epidemics, combined with historically high rates of TB (since early 1990s), raise a concern that the rates of active TB could further increase among this vulnerable population.

The purpose of the current study was to describe tuberculosis knowledge and beliefs, and experience with TB services among IDUs visiting syringe exchange programme (SEP) in Estonia.

\section{MATERIALS AND METHODS}

\section{Setting and Sample}

Data for this cross-sectional anonymous study were collected in a community based syringe exchange programme in Tallinn (capital city located in Northern Estonia). This SEP serves approximately 1,800 clients per month and distributes approximately 460,000 syringes per year (6). Recruitment was conducted during regular business hours between December 2007 and March 2008. Each client was approached for the study participation. Recruitment and questionnaire administration were executed by the full-time SEP staff. The staff received study specific training on recruitement and questionnaire administration.

Persons were eligible to participate if they were $\geq 18$ years of age, provided informed consent, were SEP clients (had the "client card" with a unique code), and were able to speak and read either Estonian or Russian.

\section{Instruments and Procedures}

All potential participants were screened for study eligibility. To avoid multiple enrollment specific markers used for identification 
were reviewed at enrollment daily. These markers included: client codes, gender, and participant age. An interviewer administered structured questionnaire. The interview was conducted privately by staff requiring approximately 20 minutes for completion. The questionnaire included: socio-demographics; history of drug use; incarceration; tuberculosis history, knowledge, services and contacts; current health concerns, and HIV. The questionnaire was created by integrating components from validated assessments (7-9). After completing all procedures, participants received a small food package as a study incentive (valued at 1.6 EUR). All participants reporting symptoms suggestive of TB were referred to local TB services. Considering that our study was anonymous, we were not able to verify the completed referral rate with the TB services.

\section{Data Management and Statistical Analysis}

The interviewers reviewed the questionnaires immediately after the interview. Completed questionnaires were collected, checked and reviewed again for inaccuracies by supervisors on a daily basis. All data were double entered. The data sets were compared to detect and correct any data entry errors. Statistical analyses were performed with STATA 11.0 (StataCorp LP. College Station, TX). Descriptive statistics were used to characterize participants and tuberculosis knowledge. Associations between participant characteristics and knowledge were evaluated by using the Wilcoxon rank-sum test or Fisher exact test. Univariate and multivariable analyses were conducted to determine the variables related to better HIV (correct answer to all three knowledge questions as the reference group), TB transmission (correct answer to both TB transmission questions as the reference group) and TB treatment adherence knowledge (correct answer to all three adherence questions as the reference group). In univariate analysis these variables examined included: gender, age, ethnicity, incarceration, duration of IDU, frequency of SEP attendance in last 4 weeks, HIV status, lifetime TB history, TB contacts, and antiretroviral (ARV) treatment in last 6 months. In multivariate analysis the variables included gender, age, and variables significantly associated with knowledge indicators in univariate analysis.

\section{Ethical Committee}

The study was approved by the Tallinn Medical Research Ethics Committee (November 2007). All participants provided oral informed consent.

\section{RESULTS}

\section{Sociodemographic Characteristics and Infectious Diseases}

The total sample size was 407 . Men accounted for $79.2 \%$ of the sample $(\mathrm{n}=319)$; the mean age was 27.9 years (median $=$ 27 , range: 18-52); and mean duration of injecting drug use was 9.4 years $($ median $=5.1$; range $0-33$ ). $88.2 \%$ were of Russian nationality $(\mathrm{n}=359) .57 .6 \%$ reported high school education or more ( $\mathrm{n}=234) .47 .4 \%$ had been in prison at least once in lifetime $(\mathrm{n}=192)$. Close to half $(44.2 \%, \mathrm{n}=180)$ had national health insurance. $46.1 \%(n=182)$ had visited syringe exchange programme at least once a week in the last 4 weeks.

Close to one third $(28.6 \%, \mathrm{n}=116)$ reported having ever lived, studied and/or worked with a person with TB. Only a few $(1.7 \%$, $n=7)$ had a lifetime history of TB. $26.4 \%$ participants $(n=106)$ reported symptoms suggestive of TB (cough more than two weeks and/or blood in sputum). 52\% $(\mathrm{n}=211)$ had had a chest $\mathrm{X}$ -

Table 1. Knowledge and beliefs about tuberculosis and HIV among injecting drug users in Tallinn, Estonia, 2007

\begin{tabular}{|c|c|c|c|c|}
\hline Knowledge/belief & Description & $\begin{array}{l}\text { Correct/expected } \\
\text { answer }\end{array}$ & Number correct & $\begin{array}{l}\text { Percentage } \\
\text { correct }\end{array}$ \\
\hline \multirow{2}{*}{ TB transmission } & By breathing air someone with TB has coughed in & Yes & 378 & 93.1 \\
\hline & Through food and water & No & 98 & 24.1 \\
\hline \multirow[b]{2}{*}{ TB treatment adherence } & You can stop taking TB medication when you start feeling good & No & 274 & 67.3 \\
\hline & $\begin{array}{l}\text { If a person stops taking TB medication before time, it will be } \\
\text { harder for them to be treated }\end{array}$ & Yes & 343 & 84.3 \\
\hline TB and HIV & If a person has HIV, it is easier to get sick with TB & Yes & 370 & 90.9 \\
\hline Seriousness & People can die because of TB & Yes & 394 & 97.0 \\
\hline Drug resistance & $\begin{array}{l}\text { There are forms of TB which cannot be treated with regular } \\
\text { kinds of drugs }\end{array}$ & Yes & 299 & 73.5 \\
\hline Personal beliefs & $\begin{array}{l}\text { Do you believe that you could get TB treatment in case } \\
\text { you got sick? }\end{array}$ & Yes & 275 & 67.6 \\
\hline Availability of TB treatment & $\begin{array}{l}\text { TB diagnostics and treatment in Estonia are free of charge } \\
\text { for everybody }\end{array}$ & Yes & 161 & 39.6 \\
\hline \multirow[b]{2}{*}{ HIV } & Can a person with HIV infection look healthy? & Yes & 383 & 94.1 \\
\hline & $\begin{array}{l}\text { It is possible to reduce HIV transmission by using condoms } \\
\text { during every sexual intercourse? }\end{array}$ & Yes & 397 & 97.5 \\
\hline Availability of HIV treatment & $\begin{array}{l}\text { Do you believe that you could get HIV treatment in case } \\
\text { you got infected? }\end{array}$ & Yes & 297 & 73.3 \\
\hline
\end{tabular}


ray within last 3 years before study participation. Three quarters had ever tested for HIV $(77.1 \%, \mathrm{n}=313)$, out of them one third (32.9\%, $n=103$ ) reported being HIV-infected. $22.3 \%$ of those HIV-positive reported receiveing antiretroviral treatment in the last six months $(\mathrm{n}=23)$.

\section{TB and HIV Knowledge}

TB and HIV knowledge data are presented in Table 1. Majority of participants $(93 \%)$ recognized correctly that TB is air-borne infection and that HIV is a risk factor for TB (91\%).

Univariate analysis showed that the frequency of correctly answering all three HIV knowledge questions was significantly associated with education ( $92 \%$ among those with 12 or more years of formal education vs. $86 \%$ among those with less education; $\mathrm{p}=0.04)$ and ever testing for HIV (93\% among those ever tested and $78 \%$ among those never tested). Logistic regression analysis confirmed the last association (AOR 3.8; 95\% CI: 1.93-7.33).

Correctly answering all three TB transmission knowledge questions was significantly associated with imprisonment $(29 \%$ among those who had ever been in prison vs. $19 \%$ among those who had not; $\mathrm{p}=0.02)$ and frequency of visiting SEP in the last four weeks (32\% among those who had visited more than once a week vs. $17 \%$ among who had visited more than once a week; $\mathrm{p}=0.001)$. Logistic regression analysis confirmed the last association (AOR 2.16; 95\% CI: 1.32-3.51).

Correctly answering all three TB treatment adherence questions was significantly associated with education (51\% among those with 12 or more years of formal education vs. $38 \%$ among those with less; $\mathrm{p}=0.01)$ and HIV infected persons on ARV treatment $(83 \%$ among those who had had ARV last six months vs. $43 \%$ among those who had not; $\mathrm{p}<0.001)$. Logistic regression analysis showed that both education (AOR 1.59; 95\% CI: 1.04-2.42) and ARV treatment (AOR 6.05; 95\% CI: 1.99-18.38) were independently associated with correct knowledge of TB treatment adherence.

\section{DISCUSSION}

Our study identified gaps in knowledge that injecting drug users had of TB transmission, treatment and health care services. Basic HIV knowledge, on the other hand, was very high. Historically, harm reduction programmes (syringe exchange and substitution treatment for opioid injectors) in Estonia were scaled up in the framework of the Global Fund to Fight AIDS, Tuberculosis and Malaria (GFATM) programme (2003-2007), its main focus having been prevention of HIV (6). Since the end of the GFATM programme, the funding has been provided through the state budget in the farmework of National HIV and AIDS Strategy for 2006-2015 (6). In Estonia, TB has not been systematically approached in harm reduction setting and there has not been tuberculosis education for the general population in recent years.

Only $44 \%$ of the participants reported having national health insurance, compared to the general population where the vast majority ( $\sim 94 \%$ ), including children and the elderly, is covered by the compulsory health insurance scheme. Estonian health insurance is a social insurance, relying on the principle of solidarity: the Estonian Health Insurance Fund covers the cost of health services required in case of illness regardless of the amount of social tax paid by the person concerned. Uninsured people, representing $6 \%$ of the population consist mainly of low-income men who either are long-term unemployed or work in the informal sector (6). Certain disease prevention and health promotion activities (including HIV and TB related health care services) are free of charge for all people, including those who do not have national health insurance (6). Previous research has also found that age restrictions, user fees, and a lack of confidentiality were not common when accessing harm-reduction programmes in Estonia (10). However, limited knowledge of this and limited coverage with health insurance may pose a barrier in accessing general health care and TB services, and therefore, delay diagnosis and treatment (6).

The relatively good knowledge of TB treatment adherence may be related to generalizing HIV information to tuberculosis. This is partly supported by the finding that TB treatment adherence knowledge was related to receiving ARV treatment. On the other hand, previous studies have discussed that also misconceptions about TB may stem from the overgeneralization of knowledge about HIV (9); therefore, TB must be paid separate attention and effective education in populations knowledgeable about HIV must highlight the differences between the two diseases.

As described elsewhere (8), knowledge was higher among those study participants who had prior contacts with different services (SEP, treatment, prison) which support the efforts to further use these venues to provide information and education to IDUs.

In Estonia, the number of patients coinfected with HIV and TB who are also injecting drug users is increasing countrywide (6). Therefore, TB prevention and education, like HIV efforts, must be integrated into harm reduction and drug treatment programmes and respective services must work in close tandem in order to facilitate early diagnosis and effective treatment of TB among injecting drug users.

\section{Limitations}

Our results may not be representative of all injecting drug users in the region because we used convenience sampling and recruited people only from one site. Recruiting only people who are already in contact with services may introduce further bias. Information obtained on TB contacts and last X-ray may be prone to recall bias. As the data collection took place in winter months, health complaints may have been related to other respiratory virus infections.

\section{Acknowledgements}

This study was financed through the National HIV and AIDS Strategy for 2006-2015. Authors are grateful to the participants for their co-operation and to the study team.

\section{REFERENCES}

1. Fears R, Kaufmann S, Ter Meulen V, Zumla A; EASAC Working Group. Drug-resistant tuberculosis in the European Union: opportunities and challenges for control. Tuberculosis (Edinb). 2010 May;90(3):182-7.

2. Perlman DC, Salomon N, Perkins MP, Yancovitz S, Paone D, Des Jarlais DC. Tuberculosis in drug users. Clin Infect Dis. 1995 Nov;21(5):1253-64.

3. Hwang LY, Grimes CZ, Beasley RP, Graviss EA. Latent tuberculosis infections in hard-to reach drug using population - detection, prevention and control. Tuberculosis (Edinb). 2009 Dec;89 Suppl 1:S41-5. 
4. Platt L, Bobrova N, Rhodes T, Uusküla A, Parry JV, Rüütel K, et al. High HIV prevalence among injecting drug users in Estonia: implications for understanding the risk environment. AIDS. 2006 Oct 24;20(16):2120-3.

5. Uusküla A, Rajaleid K, Talu A, Abel K, Rüütel K, Hay G. Estimating injection drug use prevalence using state wide administrative data sources: Estonia, 2004. Addict Res Theory. 2007;15(4):411-24.

6. Rüütel K, Trummal A, Salekešin M, Pervilhac C. HIV epidemic in Estonia: analysis of strategic information. Copenhagen: WHO Regional Office for Europe; 2011.

7. Des Jarlais DC, Perlis TE, Stimson GV, Poznyak V; WHO Phase II Drug Injection Collaborative Study Group. Using standardized methods for research on HIV and injecting drug use in developing/transitional countries: case study from the WHO Drug Injection Study Phase II. BMC Public Health. 2006 Mar 2;6:54.
8. Salomon N, Perlman DC, Friedmann P, Perkins MP, Ziluck V, Des Jarlais $\mathrm{DC}$, et al. Knowledge of tuberculosis among drug users. Relationship to return rates for tuberculosis screening at a syringe exchange. J Subst Abuse Treat. 1999 Apr;16(3):229-35

9. Wolfe H, Marmor M, Maslansky R, Nichols S, Simberkoff M, Des Jarlais $\mathrm{D}$, et al. Tuberculosis knowledge among New York City injection drug users. Am J Public Health. 1995 Jul;85(7):985-8.

10. Curth NK, Hansson LN, Storm F, Lazarus JV. Select barriers to harmreduction services for IDUs in eastern Europe. Cent Eur J Public Health. 2009 Dec;17(4):191-7.

Received June 6, 2012 Accepted in revised form October, 9, 2012 